LAWRENCE LIVERMORE N A TION AL LABORATORY
Deterministic, Nanoscale
Fabrication of Mesoscale Objects

R. Mariella Jr., M. Shirk, G. Gilmer, A. Rubenchik

September 30, 2004 
This document was prepared as an account of work sponsored by an agency of the United States Government. Neither the United States Government nor the University of California nor any of their employees, makes any warranty, express or implied, or assumes any legal liability or responsibility for the accuracy, completeness, or usefulness of any information, apparatus, product, or process disclosed, or represents that its use would not infringe privately owned rights. Reference herein to any specific commercial product, process, or service by trade name, trademark, manufacturer, or otherwise, does not necessarily constitute or imply its endorsement, recommendation, or favoring by the United States Government or the University of California. The views and opinions of authors expressed herein do not necessarily state or reflect those of the United States Government or the University of California, and shall not be used for advertising or product endorsement purposes.

This work was performed under the auspices of the U.S. Department of Energy by University of California, Lawrence Livermore National Laboratory under Contract W-7405-Eng-48. 


\title{
Deterministic, Nanoscale Fabrication of Mesoscale Objects
}

\author{
Principal I nvestigator: Raymond Mariella J r. \\ Tracking Code: 02-ERD-014 \\ Primary COW: Engineering and Manufacturing Processes
}

Annual Report Authors: R. Mariella Jr.; M. Shirk; G. Gilmer; A. Rubenchik

\section{Figure Captions}

Figure 1: SEM micrograph of vitreous carbon that has been micromachined to form a high-aspect-ratio pattern using a KrF excimer laser (248-nm wavelength pulses) via ablation trough a mask.

Figure 2: Optical profilometer image of vitreous carbon sample in Figure 1.

Figure 3: SEM micrographs of a $0.25-\mathrm{mm}$-wide "dimple" in carbon aerogel that has been micromachined via ablation with sub-ps laser pulses at $825 \mathrm{~nm}$. Note that the process caused little collateral damage in the surrounding material.

Figure 4: SEM images of nano "hairs" and nanoparticles, formed collatorally, during high-power ablation of carbon aerogel using fs laser pulses at $825 \mathrm{~nm}$ and $1 \mathrm{KHz}$ repetition rate.

Figure 5: Optical Profilometer image and line-out showing the uniformity that we were able to achieve in micromachining a trench, via oxygen-ion-beam ablation, in vitreous carbon. Conditions: $3 \mathrm{keV}, 5-\mu \mathrm{A}$ oxygen-ion beam, constant-speed, linear scan of substrate.

Figure 6: Optical Profilometer image and line-outs showing the uniformity that we were able to achieve in micromachining a "field of moguls", via oxygen-ion-beam ablation, in vitreous carbon. Conditions: $3 \mathrm{keV}, 5-\mu \mathrm{A}$ oxygen-ion beam, scan of substrate on a $x-y$ stage moving with sinusoidally-modulated speed.

\section{Annual Report Text:}

Neither LLNL nor any other organization has the capability to perform deterministic fabrication of $\mathrm{mm}$-sized objects with arbitrary, $\mu \mathrm{m}$-sized, 3-dimensional features with 20-nmscale accuracy and smoothness. This is particularly true for materials such as high explosives and low-density aerogels. For deterministic fabrication of high energy-density physics (HEDP) targets, it will be necessary both to fabricate features in a wide variety of materials as well as to understand and simulate the fabrication process.

We continue to investigate, both in experiment and in modeling, the ablation/surfacemodification processes that occur with the use of laser pulses that are near the ablationthreshold fluence. During the first two years, we studied ablation of metals, and we used subps laser pulses, because pulses shorter than the electron-phonon relaxation time offered the most precise control of the energy that can be deposited into a metal surface. The use of subps laser pulses also allowed a decoupling of the energy-deposition process from the ensuing movement/ablation of the atoms from the solid, which simplified the modeling. We investigated the ablation of material from copper, gold, and nickel substrates. We combined 
the power of the 1-D hydrocode "HYADES" with the state-of-the-art, 3-D molecular dynamics simulations "MDCASK" in our studies.

For FY04, we have stretched ourselves to investigate laser ablation of carbon, including chemically-assisted processes. We undertook this research, because the energy deposition that is required to perform direct sublimation of carbon is much higher than that to stimulate the reaction $2 \mathrm{C}+\mathrm{O}_{2}=>2 \mathrm{CO}$. Thus, extremely fragile carbon aerogels might survive the chemically-assisted process more readily than ablation via direct laser sublimation. We had planned to start by studying vitreous carbon and move onto carbon aerogels. We were able to obtain flat, high-quality vitreous carbon, which was easy to work on, experimentally and relatively easy to model. We were provided with bulk samples of carbon aerogel by Dr. J oe Satcher, but the shop that would have prepared mounted samples for us was overwhelmed by programmatic assignments. We are pursuing aligned carbon nanotubes, provided to us by colleagues at NASA Ames Research Center, as an alternative to aerogels. Dr. Gilmer started modeling the laser/thermally accelerated reactions of carbon with $\mathrm{H}_{2}$, rather than $\mathrm{O}_{2}$, due to limited information on equation of state for $\mathrm{CO}$.

We have extended our molecular dynamics models of ablation to include carbon in the form of graphite, vitreous carbon, and aerogels. The computer code has features that allow control of temperature, absorption of shock waves, and for the ejection of material from the computational cell. We form vitreous carbon atomic configurations by melting graphite in a microcanonical cell at a temperature of about 5000K. Quenching the molten carbon at a controlled rate of cooling yields material with a structure close to that of the vitreous carbon produced in the laboratory. To represent the aerogel, we have a computer code that connects "graphite" rods to randomly placed points in the 3-D computational cell. Ablation simulations yield results for vitreous carbon similar to our previous results with copper, usually involving the transient melting of the material above the threshold energy density. However, some fracturing in the solid regions occurs in this case, but was never observed in copper. These simulations are continuing, together with studies of the reaction of hydrogen with vitreous graphite at high temperatures. These reactions are qualitatively similar to that of oxygen with the carbon atoms at the surface, and the simulations should provide insight into the applicability of the use of chemical reactions to shape the surfaces of aerogels.

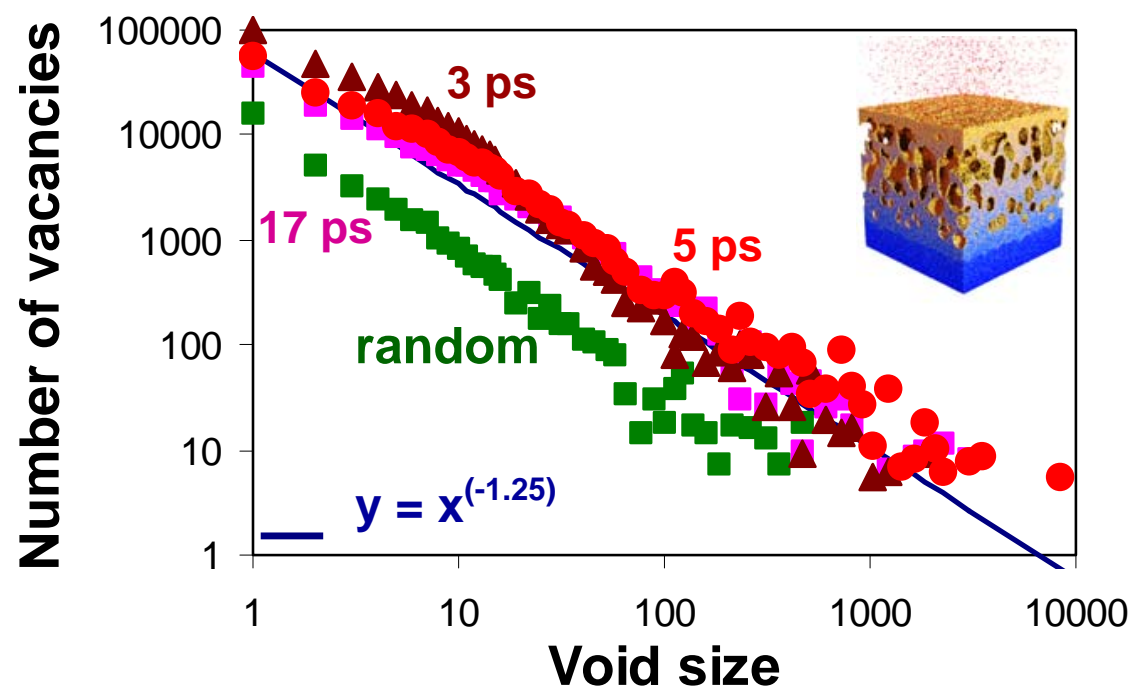


Voids nucleate during the initial phases of laser ablation and subsequently expand and coalesce, and this process releases most of the ablated material from the target. An understanding of surface roughening during ablation requires a detailed model of void formation and expansion. Our molecular dynamics models of materials such as carbon and of metals are ideal for such a study. This figure shows void sizes calculated by tracing connected vacant sites. Elapsed times after the laser pulse are indicated on the figure, and data for a random void distribution is shown. The similarity to the cluster size distribution in a random array of vacancies suggests that there is little or no barrier to void formation under ablation conditions, and this holds through much of the time period where the voids are coarsening.

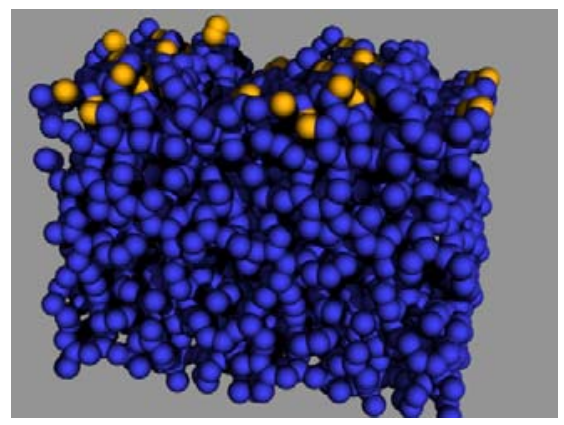

We are modeling the interaction of $\mathrm{H}$ with vitreous carbon using molecular dynamics potentials that have been developed for hydrocarbons. This figure shows a slab of vitreous carbon (blue spheres) interacting with hydrogen (yellow) at the top surface. These reactions are similar to those between oxygen and carbon, and provide insight for our experiments.

Since Dr. Siekhaus already used molecular-beam studies of a heated carbon surface reacting with a beam of $\mathrm{O}_{2}$, we had an estimate of desired temperatures (2000K) and expected reaction probabilities ( $\max =10 \%$ ). Early modeling suggested a pronounced anisotropy in the strength of interaction of $\mathrm{nm}$-diameter fibers with linearly-polarized light, and we have obtained samples of aligned carbon nanotubes from our collaborators at NASA in order to study this, experimentally.

We modeled the interaction of laser radiation with low density aerosol. Due to the low density, the penetration depth is much longer then light wavelength. The aerogel can be modeled as a network of carbon strings (nanotubes) with diameter much smaller then the light wavelength. We demonstrated that the light absorption is sensitive to the mutual orientation of the carbon string and electric field. The absorption of light with electric field parallel to the string for 1- $\mu \mathrm{m}$ light is about 60 times higher than in the situation with electric field normal to the string.

This effect greatly increases the ablation rate. Laser field penetrating in the aerogel explodes the points where electric field is parallel to the strings, breaking the string network and ejecting a lot of possibly-undamaged material. This is more efficient removal mechanism than traditional dense material evaporation and some experiments confirmed the enormously high aerogel ablation rate which was observed by Norris and co-workers (J . Non-Cryst. Solids, 281 (2001) pp 39-47).

When this project began, we had hoped to investigate the use of ion beams for surface micromachining. Dr. Siekhaus forged a collaboration with colleagues at LBL and the results of his efforts for vitreous carbon are illustrated in Figures 5 and 6.

\title{
FY04 Conference Presentations and Publications
}

\author{
Record of Invention IL-11139 \\ "Apparatus and Method of Micro-Polishing Materials" \\ Provisional Patent Filed, May 30, 2003
}




\section{Conference Talks}

Mesoscale Laser Processing using Excimer and Short-pulse Ti:Sapphire Lasers (M702)

ICALEO ${ }^{\circledR} 2003$

Oct. 13-16, 2003

Jacksonville, Florida USA

"MEMS-based sensor systems", UCRL-CONF-204129, R. Mariella J r., NNSA Futures Conference, Crystal City VA, May 2004

$3^{\text {rd }}$ International Conference: Computational Modeling and Simulation of Materials, Acireale, Sicily, Italy, May 30-June 4, 2004, Short-Pulse Laser Ablation Simulated by Molecular Dynamics, Void Nulceation and Cluster Ejection, G. H. Gilmer*, M. D. Shirk, A. M. Rubenchik, L. Zepeda-Ruiz, R. P. Mariella, T. Diaz de la Rubia, Invited Talk.

DOE-NSET Workshop on Artificially Structured Nanomaterials: Formation and Properties, Gatlinburg, TN, Oct. 13-15, 2003, Thin Film Deposition and Manipulation of Surfaces using Laser Beams: Atomistic Modeling, G. H. Gilmer, Invited Talk.

WORKSHOP I: Fundamental Physical Issues in Nonequilibrium Interface Dynamics, Center for Scientific Computation and Mathematical Modeling, University of Maryland, Oct. 20-24, 2003, Laser Ablation and the Deposition of Metals: Large-Scale Molecular Dynamics Simulations, G. H. Gilmer, Invited Talk.

2nd International Workshop on Strength and Fracture, Berkeley Marina, California, January 7-9, 2004, Short-pulse laser ablation of metals: Large-scale molecular dynamics simulations, G.H. Gilmer, Invited Talk.

American Physical Society March Meeting, Montreal, Canada, March 22-26, 2004, Short-pulse laser ablation of metals: large-scale molecular dynamics simulations. G. H. Gilmer, M. D. Shirk, A. M. Rubenchik, L. Zepeda-Ruiz, R. P. Mariella Jr, B. Sadigh, S. L. Baker, E. Bringa, Contributed Talk.

Materials Research Society Spring Meeting, San Francisco, CA, April 12-16, 2004, Void Nulceation and Cluster Ejection during Short-Pulse Laser Ablation: Large- Scale Molecular Dynamics Simulations, G. H. Gilmer, M. D. Shirk, A. M. Rubenchik, R. P. Mariella, L. Zepeda-Ruiz, S. L. Baker, B. Sadigh, Contributed Talk.

Directorate Review Committee, LLNL, May 5-7, Laser Shaping of Materials: LargeScale Molecular Dynamics Simulations, G. H. Gilmer, M. D. Shirk, A. M. Rubenchik, R. P. Mariella Jr, A. M. Komashco, S. L. Baker, B. Sadigh, B. C. Stuart, M. A. Duchaineau, and T. Diaz de la Rubia; Poster.

The Third Annual Ann Arbor Symposium on Relativistic and Sub-Relativistic Intensity Lasers and their Applications, Ann Arbor, MI, June 3 \& 4, Ben Torralva \& George Gilmer Modeling the Damage Threshold, Invited Talk 
Manuscript submitted to Science

“Laser Ablation of Metals: Large-Scale Atomistic Simulations and Experiments"

G. H. Gilmer, M. D. Shirk, A. M. Rubenchik, R. P. Mariella, A. M. Komashko,

S. L. Baker, B. Sadigh, M. A. Duchaineau, and T. Diaz de la Rubia;

Manuscripts in preparation

"Processing of dielectrics with ultrashort laser pulses", Rubenchik and Shirk

“Design and Implementation of a High-precision, Ultrashort Pulsed Laser

Milling Machine", Shirk and Rubenchik 


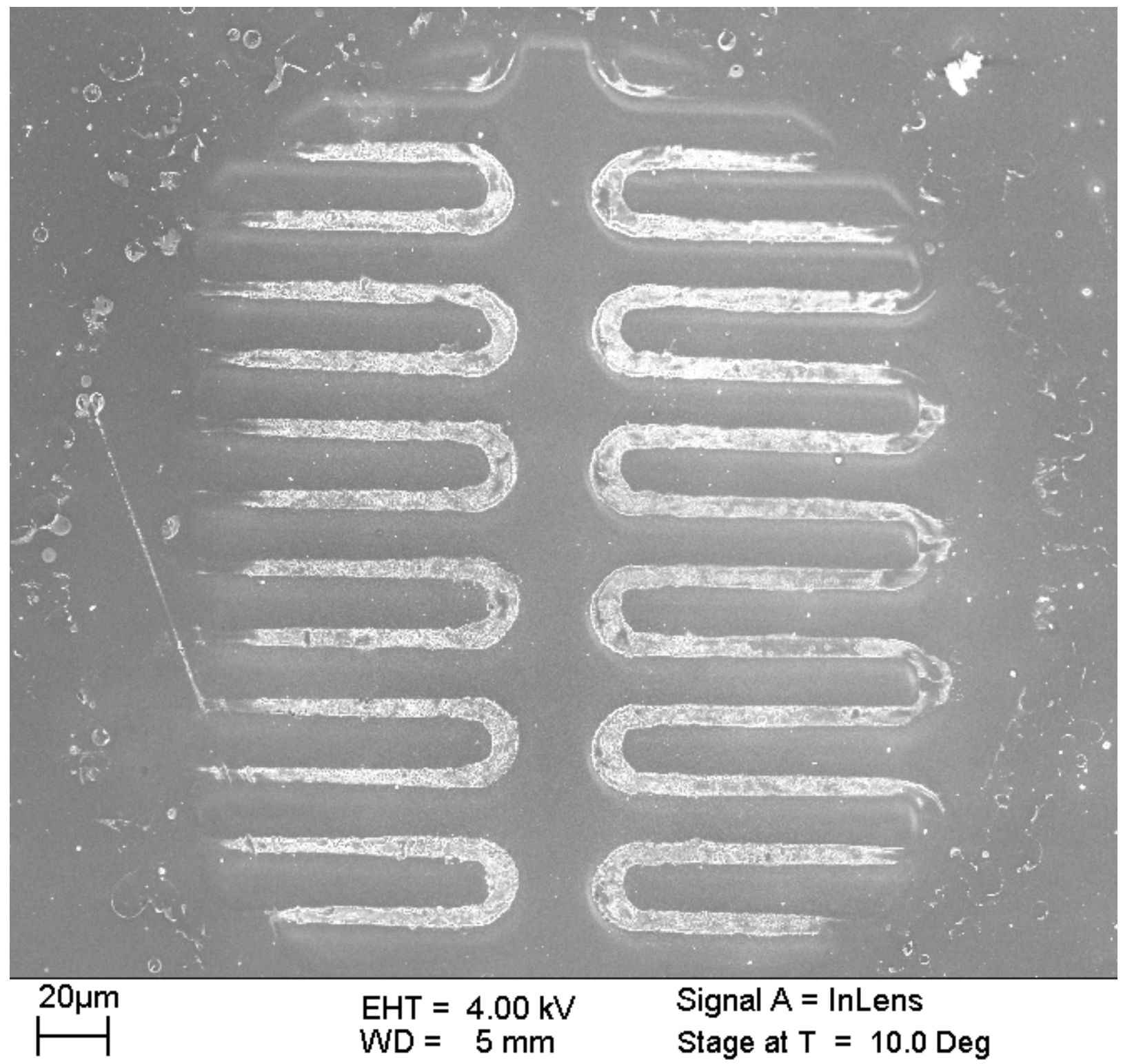

Figure 1. 


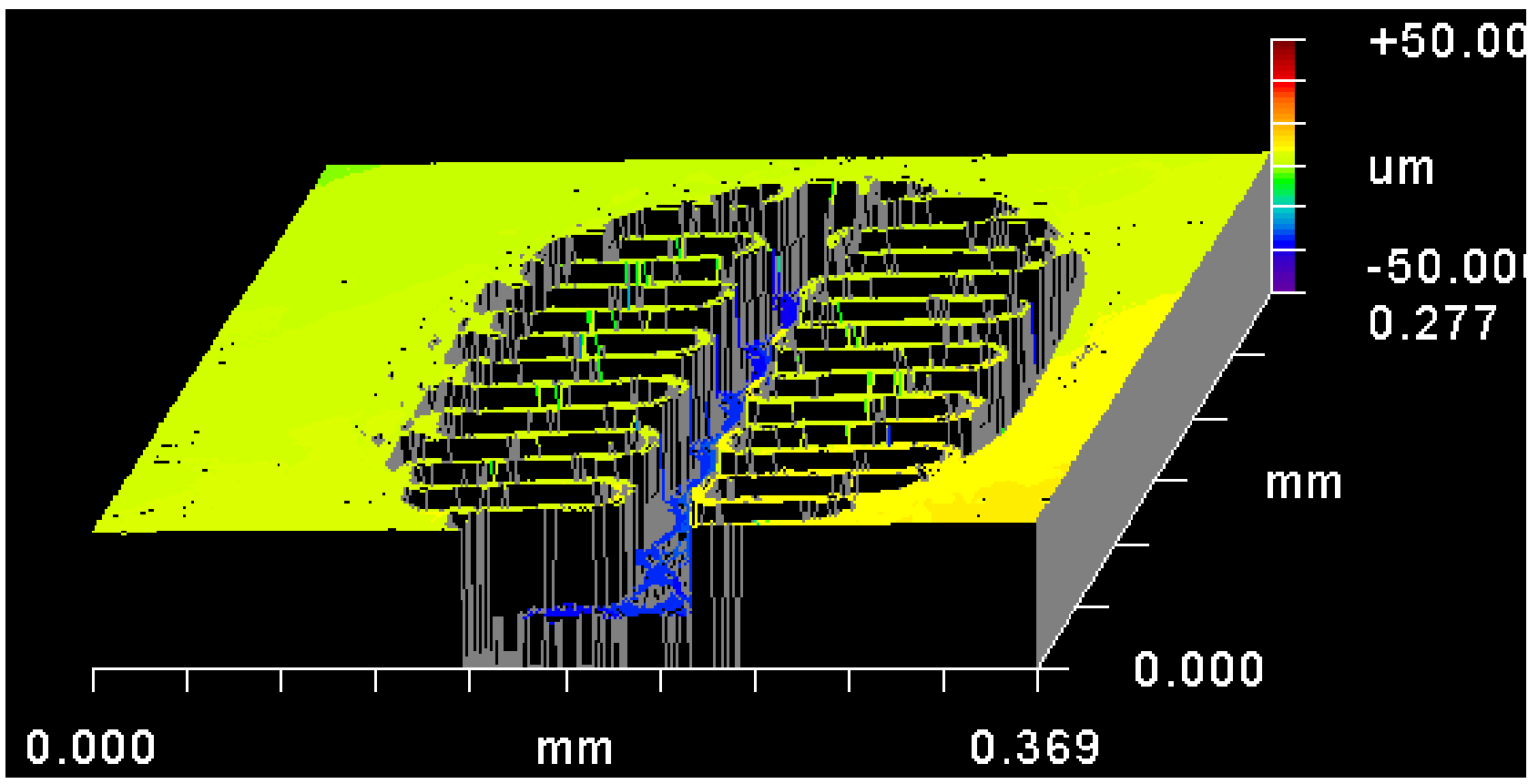

Figure 2 
TIFF (Uncompressed) decompressor are needed to see this picture.

Fig. 3. 0.25-mm wide dimple in carbon aerogel, fabricated via 

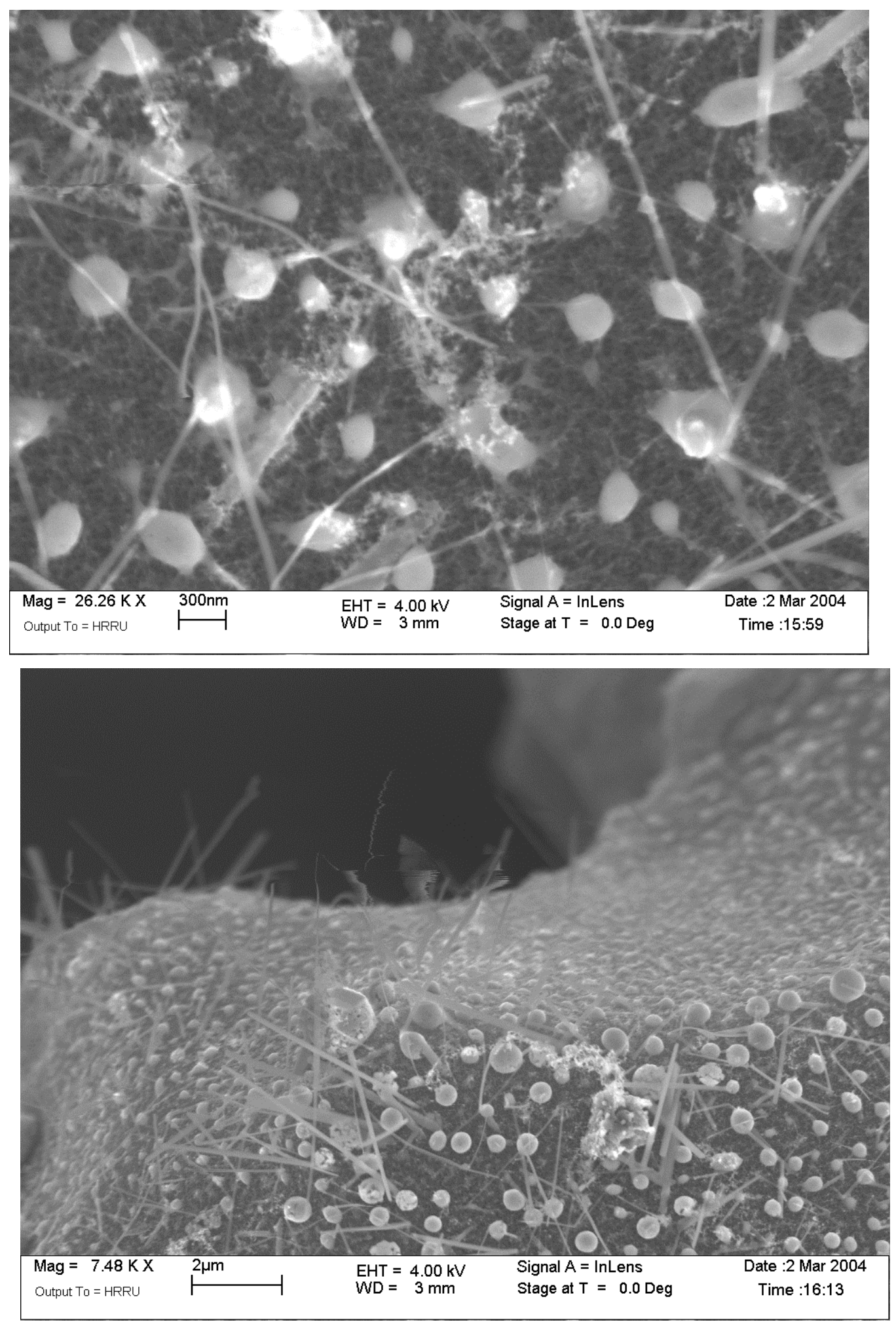

Figure 4. 


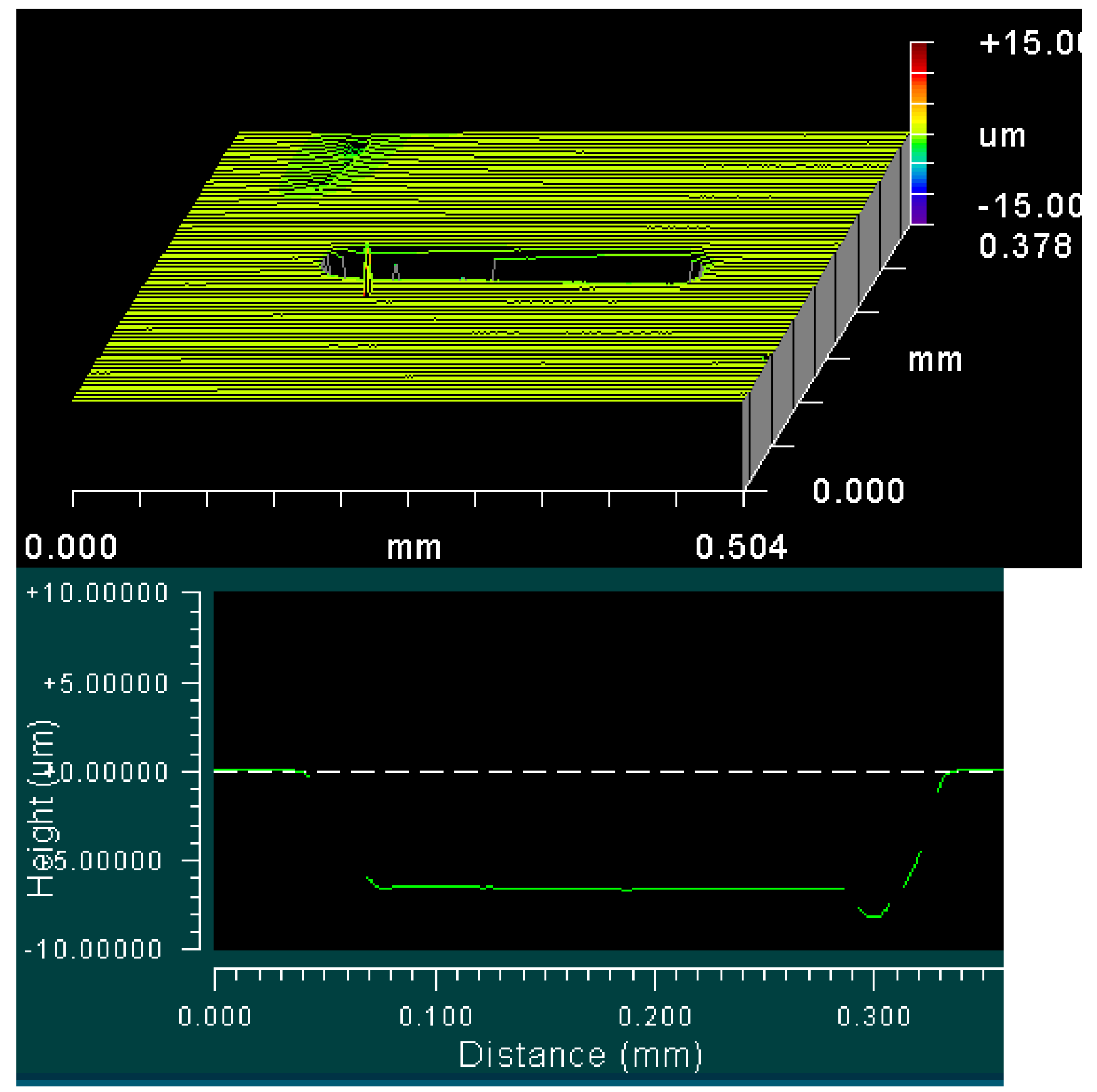

Fig 5. 

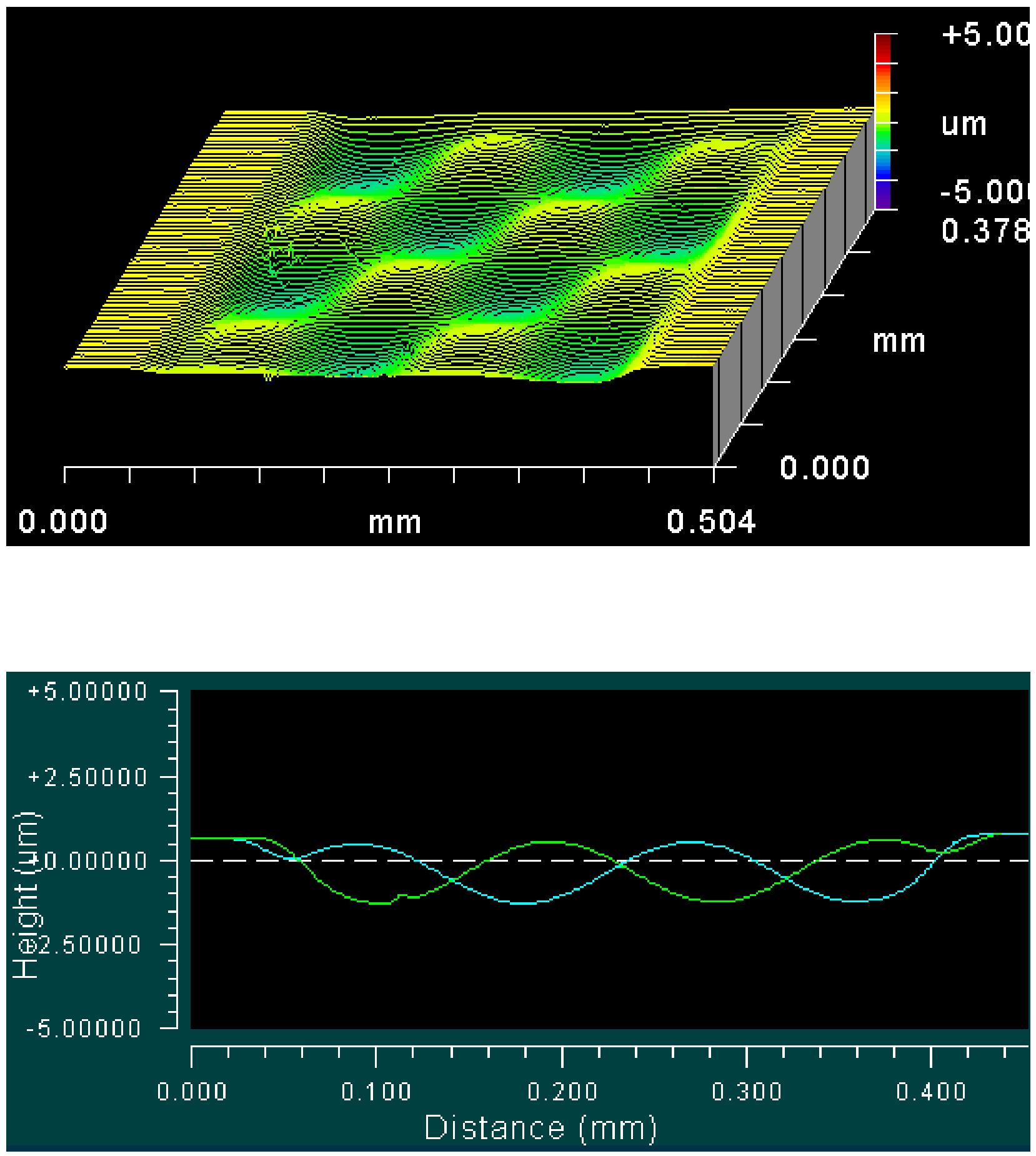

Figure 6. 